\title{
CLASIFICACIÓN GEOMECÁNICA Y ANÁLISIS ESTABILIDAD DE TALUDES DEL MACIZO ROCOSO CORIS, CARTAGO, COSTA RICA
}

\author{
Maureen Carrillo, Lepolt Linkimer, Albán Rodríguez \& Héctor Zúñiga \\ Escuela Centroamericana de Geología, \\ Apdo. 2-14, 2060 Universidad de Costa Rica
}

\section{INTRODUCCIÓN}

El método Rock Mass Rainting (RMR) para la clasificación de macizos rocosos fue desarrollado por Bieniawski (1972). Este método permite, de forma sencilla, estimar la calidad del macizo rocoso, mediante la cuantificación de parámetros de fácil medición, los cuales se establecen en el campo de manera rápida y con costos económicos mínimos. El método RMR incluye los siguientes parámetros: resistencia a la compresión uniaxial de la roca, Rock Quality Designation (RQD), espaciamiento de discontinuidades, condición de las discontinuidades, condición del agua subterránea y orientación de las discontinuidades.

Con el valor del RMR es posible establecer algunas propiedades geotécnicas preliminares del macizo, para analizar la estabilidad del talud del frente de explotación actual.

El sistema Q, fue propuesto por Barton et al. (1974), basándose en una gran cantidad de casos tipo de estabilidad en excavaciones subterráneas, siendo su principal propósito establecer un índice para determinar la calidad del macizo rocoso en túneles. El sistema Q incluye parámetros como el índice de calidad de la roca (RQD), número de sistemas de fisuras $\left(\mathrm{J}_{\mathrm{n}}\right)$, rugosidad de las fisuras $\left(\mathrm{J}_{\mathrm{r}}\right)$, alteración de las fisuras $\left(\mathrm{J}_{\mathrm{a}}\right)$, factor de reducción por agua en las fisuras $\left(\mathrm{J}_{\mathrm{w}}\right)$, y el factor de reducción por esfuerzos (SRF). El valor numérico del índice $\mathrm{Q}$ se obtiene a partir de la siguiente ecuación:

$$
\mathrm{Q}=\left(\mathrm{RQD} / \mathrm{J}_{\mathrm{n}}\right) *\left(\mathrm{~J}_{\mathrm{r}} / \mathrm{J}_{\mathrm{a}}\right) *\left(\mathrm{~J}_{\mathrm{w}} / \mathrm{SRF}\right) .
$$

Este artículo involucra el estudio de las propiedades físicas y mecánicas más relevantes del macizo rocoso de la Formación Coris, expuesto en la mina, propiedad de la empresa Sílice de Costa Rica S.A. (SICORSA). La mina se localiza a 250 metros al NE del poblado de Coris, provincia de Cartago, en las coordenadas $205,9 \mathrm{~N}$ y $537,7 \mathrm{~W}$, de la hoja topográfica Istarú, escala 1:50 000 del Instituto Geográfico Nacional (Fig. 1)

\section{GEOLOGÍA REGIONAL}

En la región de Coris afloran rocas pertenecientes a tres unidades estratigráficas diferentes, las cuales representan los últimos estadíos de la evolución de la cuenca del Valle Central. Las principales características de estas formaciones geológicas son las siguientes:

Formación San Miguel: Corresponde con al menos $200 \mathrm{~m}$ de calcarenitas de grano 


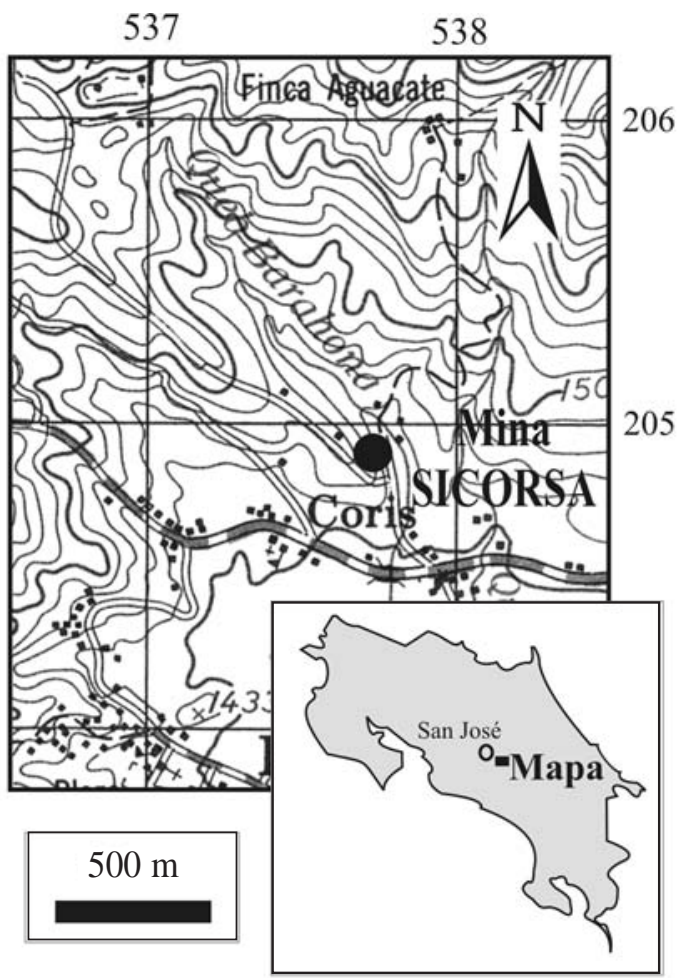

Fig. 1: Ubicación del sitio de estudio.

medio y calizas bioclásticas, asociadas con una sedimentación marina somera, con leve o sin influencia volcánica (Badilla et al., 1999). Su edad es Mioceno Medio (Carballo, 1978).

Formación Coris: Consiste de sedimentos del Mioceno Inferior terminal al Mioceno Superior (Fischer \& Franco, 1979), principalmente areniscas cuarzosas, areniscas volcaniclásticas, lodolitas y horizontes de carbón, relacionadas con un ambiente marino somero o de barras arenosas, que fue acompañado de eventos volcánicos y de fuerte erosión (Badilla et al., 1999).

Formación Aguacate: Incluye brechas volcaniclásticas en variedad de granulometría, tobas, lavas y rocas hipoabisales, relacionadas con la actividad volcánica que prevalece a partir del Mioceno Superior terminal (Badilla et al., 1999). La unidad inferior, denominada Formación La Cruz, incluye coladas de basalto y leucobasalto, con tobas intercaladas de colores rojos y violáceos (Denyer \& Arias, 1991).

\section{GEOLOGÍA LOCAL}

El macizo rocoso en estudio está constituido por areniscas cuarzosas de la Formación Coris, de color crema y café, con un tamaño de grano fino a medio, bien sorteadas y compuestas esencialmente de cuarzo. Estas rocas poseen muy poca matriz, la cual está compuesta principalmente de cuarzo de grano fino, arcillas y óxidos de hierro. También presenta minerales como magnetita y goethita causantes de la oxidación de la superficie y de las bandas de óxidos de hierro de color rojizo y lila. Los granos son subangulares a subredondeados, con contacto longitudinal y puntual y con cemento silíceo. La roca no presenta estructuras sedimentarias definidas, ni fósiles, aunque ocasionalmente presenta algunas huellas de bioturbación, con estructuras rellenas del mismo sedimento (SICORSA, 1999).

\section{CLASIFICACIÓN DEL MACIZO ROCOSO}

De acuerdo con la clasificación y propiedades índice de las rocas, el macizo rocoso estudiado posee una textura clástica, con cemento débil. A continuación se describen las principales características del macizo rocoso, de acuerdo con la clasificación RMR (Bieniawski, 1972) y con el sistema Q (Barton et al., 1974).

\section{Rock Mass Raiting (RMR)}

Resistencia a la compresión uniaxial de la roca

Se realizó un ensayo de comprensión uniaxial a cuatro muestras de roca recolectadas en el macizo rocoso. El valor promedio para el material arenoso es de 7,7 $\mathrm{MPa}$, lo que corresponde con una roca de mala calidad y con una valoración de 2.

\section{Indice de Calidad de la Roca, RQD}

El RQD se ha estimado midiendo la cantidad de discontinuidades en $1 \mathrm{~m}^{2}$ del talud. Su valor fue determinado usando la fórmula empírica propuesta por Bieniawsky (1989): 
$\mathrm{RQD}=115-3,3\left(\mathrm{~J}_{\mathrm{v}}\right)$

En donde:

$\mathrm{J}_{\mathrm{v}}=$ número de discontinuidades en $1 \mathrm{~m}^{2}$

$\mathrm{RQD}=115-3,3(28)$

$\mathrm{RQD}=22,6$.

De acuerdo con la clasificación de Deere (1964), la calidad de la roca se considera muy mala $(<25 \%)$ y su valoración es de 5 .

\section{Espaciamiento de las discontinuidades}

Se midieron tres sistemas de discontinuidades (denominados 1, 2 y 3) que corresponden únicamente con diaclasas. El rumbo y espaciamiento de las discontinuidades se resumen en el cuadro 1.

La valoración del espaciamiento entre las discontinuidades es de 9 , considerando el sistema 3 como el más desfavorable para la estabilidad del talud (ya que se presenta cada $30 \mathrm{~cm}$ en el macizo).

\section{Condición de las discontinuidades}

El cuadro 2, contiene las características de los sistemas de diaclasas observados.

\section{Condiciones del agua subterránea}

El macizo rocoso se presenta totalmente seco, por lo tanto su valoración es de 10, según la clasificación propuesta por Deere en 1964.

\section{Clasificación del macizo rocoso, RMR}

La valoración total del macizo rocoso es de 40 y se desglosa en el cuadro 3. Este valor corresponde con un macizo rocoso de categoría IV, es decir, de mala calidad, puede considerarse un tiempo medio de sostén de 5 horas para un trecho de 15 metros (en túneles), cohesión de 100 a 200 $\mathrm{kPa}$ y un ángulo de fricción de $15^{\circ}$ a $25^{\circ}$.

Cuadro 1

Sistemas de discontinuidades en el macizo rocoso

\begin{tabular}{ccccc}
\hline Sistema & Rumbo & Dirección de buzamiento & Ángulo de buzamiento & Espaciamiento de discont. (m) \\
\hline 1 & $\mathrm{~N} 55^{\circ} \mathrm{W}$ & $\mathrm{N} 35^{\circ} \mathrm{E}$ & $52^{\circ}$ & 1,63 \\
2 & $\mathrm{~N} 53^{\circ} \mathrm{E}$ & $\mathrm{S} 37^{\circ} \mathrm{E}$ & $82^{\circ}$ & 1,45 \\
3 & $\mathrm{~N} 59^{\circ} \mathrm{W}$ & $\mathrm{S} 31^{\circ} \mathrm{W}$ & $68^{\circ}$ & 0.30 \\
\hline
\end{tabular}

Cuadro 2

Características de las discontinuidades del macizo rocoso

\begin{tabular}{lllll}
\hline Parámetro & Sistema 1 & Sistema 2 & Sistema 3 & Valoración \\
\hline Long. discont $(\mathrm{m})$ & $3-10$ & $3-10$ & $3-10$ & 2 \\
Apertura (mm) & 5 & $>5$ & $>5$ & 0 \\
Rugosidad & Suave & Leve & Leve & 1 \\
Relleno & Ninguno & Ninguno & Ninguno & 6 \\
Meteorización & Leve & & Leve & 5 \\
\hline TOTAL & & & 14 \\
\hline
\end{tabular}


Cuadro 3

Valoración total del macizo rocoso

Resistencia a la compresión uniaxial de la roca 2 RQD

Espaciamiento de las discontinuidades

Longitud de las discontinuidades

Apertura

Rugosidad

Relleno

Meteorización

Condiciones del agua subterránea

TOTAL (RMR)

\section{Orientación de las discontinuidades}

El rumbo del talud es $\mathrm{S} 70^{\circ} \mathrm{E}$, inclinado hacia el SW. En vista de esto, el sistema 1, no es desfavorable para la estabilidad del talud, ya que su buzamiento es hacia el NE (dirección contraria al talud). Sin embargo, los sistemas 2 y 3 , se consideran desfavorables, debido a que buzan hacia el SE y SW, respectivamente.

El ajuste por la orientación de las fisuras, se hace considerando que ambos sistemas son medianamente desfavorables $(-25)$ o desfavorables (-50), de manera que en cada caso, debe sustraerse a la valoración total del RMR, una cantidad de 25 y 50 respectivamente, como se muestra en el cuadro 4.

De esta manera, el RMR, caracterizaría un macizo rocoso de categoría $\mathrm{V}$, el cual posee las siguientes características:

A) se considera muy malo.

B) tiene un tiempo medio de sostén de 10 minutos para un trecho de 0,5 m (en túneles).

C) posee una cohesión < $100 \mathrm{kPa}$ y ángulo de fricción $<15^{\circ}$.

\section{El sistema $\mathbf{Q}$}

Índice de calidad de la roca $(R Q D)$

Como se mencionó anteriormente, el macizo rocoso posee un RQD de 22,6.

Número de sistemas de fisuras $\left(J_{n}\right)$

Se midieron tres sistemas de discontinuidades, que corresponden únicamente con diaclasas. Esto representa una valoración de 9.

\section{Rugosidad de las fisuras $\left(\mathrm{J}_{r}\right)$}

El cuadro 2 muestra las características de las discontinuidades observadas en el macizo rocoso. En el caso más crítico, la rugosidad es suave, lo que corresponde con una valoración de 2 .

\section{Alteración de las fisuras $\left(J_{a}\right)$}

Las fisuras se caracterizan por presentar partículas arenosas, roca desintegrada, exenta de arcilla. Esto corresponde con una valoración de 4.

Factor de reducción por agua en las fisuras $\left(J_{w}\right)$

Como se mencionó anteriormente, el macizo rocoso se presenta totalmente seco, por lo tanto su valoración es de 1 .

Factor de reducción por esfuerzos (SRF)

La valoración de este factor es de 2.5, ya que el macizo rocoso se caracteriza por presentar zonas de debilidad aisladas con arcilla o roca químicamente desintegrada (profundidad de excavación mayor a $50 \mathrm{~m}$ ).

Cuadro 4

Ajuste de la valoración por orientación de las fisuras

\begin{tabular}{lccc}
\hline Condición de la orientación de las fisuras & Ajuste en la valoración & Valor del RMR & RMR ajustado \\
\hline Medianamente desfavorable & -25 & 40 & 15 \\
Desfavorable & -50 & 40 & -10 \\
\hline
\end{tabular}


Valor de $Q$

Sustituyendo los valores: RQD $=22,6 ; \mathrm{J}_{\mathrm{n}}$ $=9 ; \mathrm{J}_{\mathrm{r}}=2 ; \mathrm{J}_{\mathrm{a}}=4 ; \mathrm{J}_{\mathrm{w}}=1$ y $\mathrm{SRF}=2,5 ;$ se obtiene un valor de $Q$ de:

$$
\begin{aligned}
& \mathrm{Q}=\left(\mathrm{RQD} / \mathrm{J}_{\mathrm{n}}\right) *\left(\mathrm{~J}_{\mathrm{r}} / \mathrm{J}_{\mathrm{a}}\right) *\left(\mathrm{~J}_{\mathrm{w}} / \mathrm{SRF}\right) \\
& \mathrm{Q}=(22,6 / 9) *(2 / 4) *(1 / 2,5) \\
& \mathrm{Q}=0,50
\end{aligned}
$$

En 1976, Bieniawski propone una ecuación que se correlaciona el valor de Q con el valor teórico que se obtendría utilizando el sistema RMR. De esta forma se tiene que RMR $=9$ $\ln (\mathrm{Q})+44$, obteniéndose un valor de $\mathrm{RMR}=$ 37,76 , y luego de aplicar los ajustes por orientación de las discontinuidades, el valor final de RMR oscilaría entre 12,76 y - 12,24, correspondiendo este resultado con un macizo de categoría V cuyas características ya han sido mencionadas.

\section{ANÁLISIS DE ESTABILIDAD DEL TALUD}

Para el análisis de la estabilidad del talud en el frente de mina, se utilizó el programa de cómputo PC-Slope V 6,0 para ambiente DOS. Las propiedades mecánicas de la roca necesarias para el modelo, se asumen de las propuestas por el método RMR para un macizo de categoría V.

$\mathrm{El}$ análisis se realizó asumiendo las siguientes condiciones:

- Una superficie de ruptura circular, considerando que la interacción de los tres sistemas de discontinuidades induce un comportamiento homogéneo al macizo. Se excluye la posibilidad de una superficie de ruptura a lo largo de algún plano de discontinuidad.

- Un talud vertical, con una altura inicial de 7 metros.

- $\quad$ Un nivel freático no aflorante.

- Ausencia de aceleraciones inducidas por actividad sísmica.

Un talud con las condiciones propuestas posee un factor de seguridad de 2,2 (Fig. 2). Por

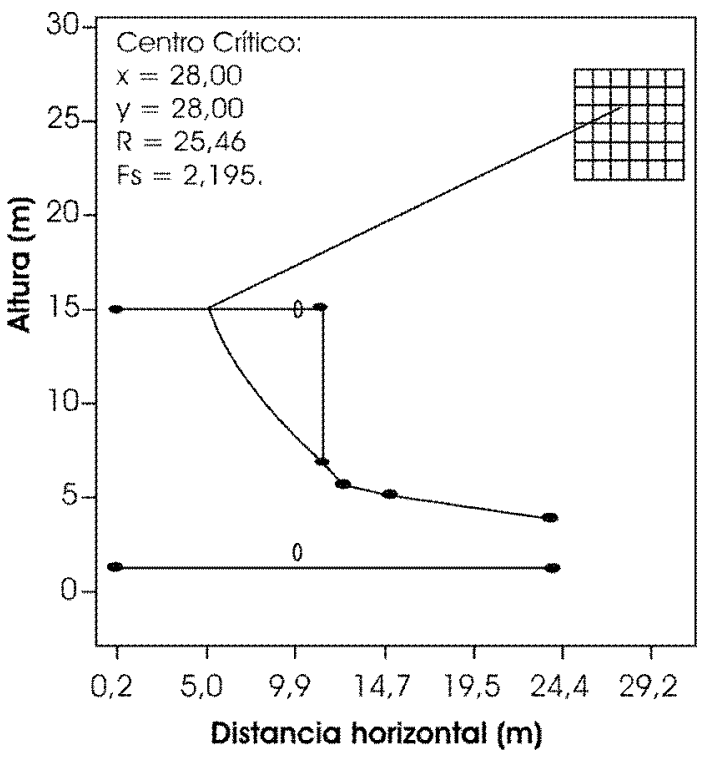

Fig. 2: Perfil del sitio de Mina SICORSA, mostrando la posible superficie de ruptura por el método simplificado de Bishop.

lo tanto, es posible diseñar taludes de mayor altura sin que ocurra un colapso del talud. Realizando varias pruebas, se determinó que el talud vertical es estable hasta una altura de 15 metros, con un factor de seguridad de 1,3, valor mínimo establecido para estabilidad en tajos.

\section{CONCLUSIONES}

El macizo rocoso estudiado se clasifica como de muy mala calidad, con un RMR $<20$, un RQD de 23 y un Q de 0,5. La orientación de los sistemas de discontinuidades y su ángulo de buzamiento, se consideran como las características más desfavorables que posee este macizo rocoso en particular.

El caso más crítico de estabilidad será cuando el talud de explotación se oriente paralelo a alguno de los sistemas de diaclasas, con lo cual, existe la posibilidad de deslizamiento a lo largo de las discontinuidades. En la mina propiedad de SICORSA, la orientación del frente norte de explotación es de $\mathrm{S} 70^{\circ} \mathrm{E}$, con inclinación al SW. Es por esta razón que los sistemas 
denominados 2 y 3 , se consideran como medianamente desfavorables o desfavorables, ya que se inclinan al $\mathrm{S} 37^{\circ} \mathrm{E}$ y al $\mathrm{S} 31^{\circ} \mathrm{W}$.

El frente de explotación actual, con una altura de $7 \mathrm{~m}$, tiene un factor de seguridad de 2,2. De acuerdo con el análisis realizado, el talud es estable hasta una altura de $15 \mathrm{~m}$, alcanzando un factor de seguridad de 1,3.

\section{REFERENCIAS}

BADILLA, E., LINKIMER, L. \& ZÚÑIGA, H., 1999: Geología de Navarro y alrededores, provincia de Cartago, Costa Rica. - 100 págs. Univ. de Costa Rica [Inf. Campaña Geológica].
BARTON, N., LIEN, R. \& LUNDE, J., 1974: Engineering classification of rock masses for the design of tunnel support. - Rock Mechanics, 6: 189-236.

CARBALlO, M., 1978: La Formación San Miguel, Mioceno Inferior, Costa Rica. - 114 págs. Univ. de Costa Rica, San José [Tesis Lic.].

DENYER, P. \& ARIAS, O., 1991: Estratigrafía de la región central de Costa Rica. - Rev. Geol. América Central, 12: 1-59.

FISCHER, R. \& FRANCO, J., 1979: La Formación Coris (Mioceno, Valle Central, Costa Rica). - Inf. Semestr. IGN, 1: 15-72.

SICORSA, 1999: Informe técnico de labores. - 43 págs. Bermejo, Cartago, Costa Rica [Inf. interno]. 